\title{
Description of laminar-turbulent transition of an airfoil boundary layer measured by differential image thermography using directed percolation theory
}

\section{Tom Wester ( $\nabla$ tom.wester@uni-oldenburg.de )}

ForWind, Institute of Physics, University of Oldenburg https://orcid.org/0000-0002-8961-7570 Joachim Peinke

ForWind, Institute of Physics, University of Oldenburg

Gerd Gülker

ForWind, Institute of Physics, University of Oldenburg

\section{Article}

Keywords: Transition, Differential Image Thermography (DIT), Directed Percolation, Boundary Layer

Posted Date: January 13th, 2022

DOI: https://doi.org/10.21203/rs.3.rs-1152211/v1

License: (c) (1) This work is licensed under a Creative Commons Attribution 4.0 International License.

Read Full License 


\title{
Description of laminar-turbulent transition of an airfoil boundary layer measured by differential image thermography using directed percolation theory
}

\author{
Tom T. B. Wester ${ }^{1,{ }^{*}}$, Joachim Peinke ${ }^{1}$, and Gerd Gülker ${ }^{1}$ \\ ${ }^{1}$ ForWind, University of Oldenburg, Institute of Physics, Oldenburg, Germany \\ *e-mail: tom.wester@uni-oldenburg.de
}

\begin{abstract}
Transition from laminar to turbulent flow is still a challenging problem. Recent studies indicate a good agreement when describing this phase transition with the directed percolation theory. This study presents a new experimental approach by means of differential image thermography (DIT) enabling to investigate this transition on the suction side of a heated airfoil. The results extend the applicability of the directed percolation theory to describe the transition on curves surfaces.

The experimental effort allows for the first time an agreement between all three universal exponents of the $(1+1) D$ directed percolation for such airfoil application. Furthermore, this study proves that the theory holds for a wide range of flows, as shown by the various conditions tested. Such a large parameter space was not covered in any examination so far. The findings underline the significance of percolation models in fluid mechanics and show that this theory can be used as a high precision tool for the problem of transition to turbulence.
\end{abstract}

Key points: Transition, Differential Image Thermography (DIT), Directed Percolation, Boundary Layer

Website summary: The transition from laminar to turbulent flow is still a not fully understood phenomenon in fluid dynamics. In the current study, we show how the $(1+1) \mathrm{D}$ directed percolation theory can be used to describe this complex flow situation in the boundary layer of an airfoil.

\section{Introduction}

The description of a laminar-turbulent transition in flows is still one of the most challenging problems in turbulence research since it was first observed in Reynolds' famous pipe flow experiment in $1883^{1}$. Until now only very few studies so far have provided a comprehensive understanding of how turbulence is induced and sustained in the system under investigation. Recent reviews on the ongoing research on wall bound transition are presented by Manneville ${ }^{2}$ and Barkley ${ }^{3}$.

To reduce the complexity, Pomeau proposed more than three decades ago to describe the complex non-linear transition phenomena of a flow as interacting oscillators ${ }^{4}$. His description is based on the subcritical bifurcation of the flow in the region of the critical Reynolds number, where the flow decides between a stable and a metastable state. This new perspective on the transition started the discussion if the process of laminar-turbulent transition may fall into one of the universality classes of the directed percolation.

Pomeau's idea is very suggestive here, since the laminarturbulent transition can be described as a so-called spatiotemporal intermittency ${ }^{5,6}$. Nevertheless, it took decades before the percolation theory was taken up again in the field of fluid dynamics, although the model has been applied successfully to other topics like epidemics and forest fire $^{7}$. Pomeau's explanation is the lack of accuracy of the experimental and numerical studies in fluid dynamics available up to that time, which renders it impossible to verify the theory ${ }^{8}$. Thus, for laminar to turbulent transition experiments, high-resolution spatio-temporal methods are required, while numerical flow simulations must be sufficiently long for conclusive statistical evaluations.

In recent years, an increasing number of studies have become possible due to steady improvements of available numerical as well as experimental resources ${ }^{1}$. The influence of the order parameter on the propagation of turbulent cells was investigated ${ }^{9,10}$. DNS simulations have checked how clusters of turbulent cells form and develop $^{11}$. The determination of turbulent cells from simulations was discussed ${ }^{12}$. In addition to these studies, low-order models for describing the transition were developed $^{13}$, sophisticated simulations were performed ${ }^{14,15}$, and the applicability of percolation theory was demonstrated in experiments ${ }^{16-20}$. More recent studies also include perturbing coherent structures and their influence on the typical result of percolation ${ }^{21}$. The high topicality of this can also be seen in current conference contributions $^{22-24}$

Most of the mentioned studies focus on generic fundamental flows, while ${ }^{20}$ presents the first application of the theory to a real-world problem. Here a transition of a laminar airfoil boundary layer flow into a laminar separation bubble is investigated using stereoscopic PIV. The study

\footnotetext{
${ }^{1}$ For an explanation of the following terminology used here, please refer to the later chapter 4 .
} 
shows a good agreement with $(1+1) \mathrm{D}$ directed percolation for this phenomenon but focuses only on one angle of attack and one velocity, leaving open the question of whether the agreement also holds when the experimental parameters, such as angle of attack or inflow velocity, are varied. Since the laminar separation bubble case is very specific, it would also be of interest whether the more often occurring natural laminar-turbulent transition of the airfoil boundary layer also falls into this universality class of percolation.

A description and determination of a boundary layer transition and its location is of great importance in fluid mechanic applications. A more precise description using a model like directed percolation could improve CFD simulations (which often do not fully resolve the transition) or a prediction of loads. Unforeseen loads often result from an increased energy input of a turbulent boundary layer into the near-surface flow. This can result in a longer-attached flow followed by a delayed and stronger flow separation. Likewise, an already detached flow can suddenly reattach due to the added energy, which leads to a sudden increase of loads. Such effects lead to rapidly changing forces, which is undesirable in most applications and must consequently be understood. Therefore, percolation theory represents an interesting approach to predict, locate, understand, and model the transition phenomenon.

For an experimental approach to this boundary layer phenomenon, the challenge is to achieve a sufficiently good resolution. However, this is a major challenge for airfoils due to their curved surface and the three-dimensional nature of the evolving boundary layer. Planar PIV measurements using light sheets quickly reach their limits, as the distance between surface and measurement volume varies. This problem is also made responsible for possible deviations in ${ }^{20}$ and must be overcome for precise analysis.

Differential image thermography (DIT) appears to be an excellent tool for gaining detailed insight into the boundary layer flow of complex geometries. Many studies have proven the DIT to have a great potential to measure the unsteady boundary layer sufficiently well ${ }^{25-27}$. The method was also applied to investigate the boundary layer flow for pitching airfoils ${ }^{28}$, and to investigate the phenomenon of dynamic stall ${ }^{29}$. Other studies focused on the boundary layer transition in rotating rotor blades on small ${ }^{30}$ and large scales ${ }^{31,32}$. This wide range of applications shows the possibilities offered by the DIT and its applicability to complex situations, despite its straightforward handling.

In the present study we will measure the laminarturbulent transition of an airfoil boundary layer for different inflow velocities and angles of attack. To provide sufficient insight into the boundary layer flow despite the curved surface, DIT is used. Based on the measured tem- perature gradients, the location of the laminar-turbulent transition is determined. In a further statistical evaluation, the $(1+1) \mathrm{D}$ directed percolation theory is applied to interpret the boundary layer state. The applicability of the theory can be confirmed for a large parameter range and is used for a more accurate localization of the transition point.

We will first present the experimental setup in Sec. 2 . This includes the wind tunnel and the thermography system. In chapter Sec. 3 the DIT results are presented followed by chapter Sec. 4, where the directed percolation theory is applied to the measured data. Finally, Sec. 5 concludes the paper.

\section{Experimental Setup}

Experiments are performed in a Göttingen type return wind tunnel of the University of Oldenburg shown in Fig. 1, which is equipped with a PID velocity control. The wind tunnel has a closed test section with a size of $0.25 \mathrm{~m} \times 0.25 \mathrm{~m} \times 2.00 \mathrm{~m}$ (height $\times$ width $\times$ length) . The test section is made of acrylic glass to allow optical access. To also allow optical access in the infrared wavelength range for the DIT measurements, a calcium fluoride window (IR transmission $>90 \%$ ) is embedded in the ceiling of the test section. The position of this window can be adjusted to optimize the camera's angle of view onto the object.

A turntable is embedded $1 \mathrm{~m}$ downstream of the nozzle in the sidewall of the test section to allow the mechanical change of the angle of attack (AoA, $\alpha$ ) of the object under investigation. A DU95-W-180 airfoil ${ }^{33}$ with a chord length of $180 \mathrm{~mm}$ is selected for the experiments. The hollow airfoil is made of aluminum and covered with a black foil to ensure a high heat capacity. During the experiments it is heated from the inside using a hot air flow. Hereby a difference of approx. $5 \mathrm{~K}$ to the equilibrium state can be achieved. This ensures a sufficiently large contrast during the measurement ${ }^{2}$.

The surface temperature of the airfoil is measured by a thermography camera of type ImageIR $8380 \mathrm{hpS}$ by Infratec. The chosen settings allow an accuracy of $20 \mathrm{mK}$ with a temporal resolution of $355 \mathrm{fps}$ using the entire sensor chip with a size of $640 \mathrm{px} \times 512 \mathrm{px}$. Due to storage limitations a total of 1775 images are acquired per data set resulting in a duration of 5 s each. The field of view (FOV) has a dimension of $107.9 \mathrm{~mm} \times 49.8 \mathrm{~mm}$ with a spatial resolution of $0.172 \mathrm{~mm} / \mathrm{px}\left(\alpha=0^{\circ}\right)$. The captured FOV is illustrated on the airfoil in Fig. 1. For the calculation of the differential images, an image of the heated airfoil is taken for each measurement series before the wind tunnel is switched on. This image then serves as a reference image for the subsequent

\footnotetext{
${ }^{2}$ The temperature difference is so small that we expect no fundamental changes in the aerodynamics on the boundary layer of the profile.
} 


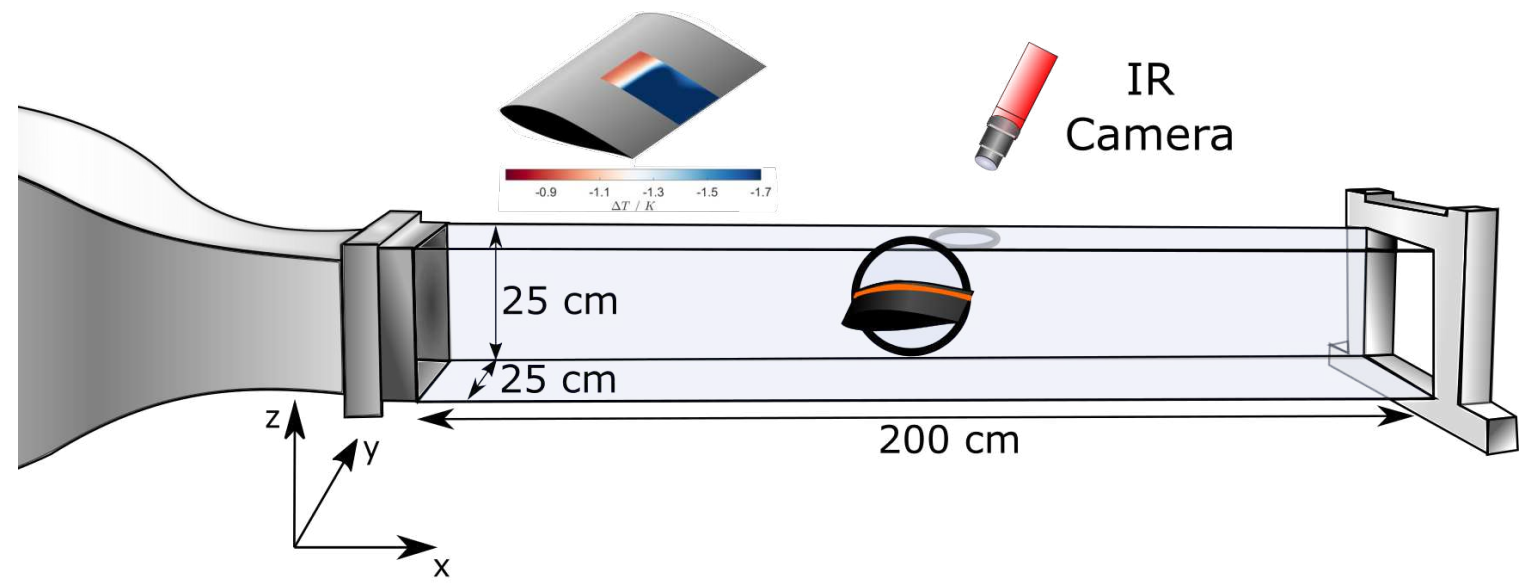

Figure 1. Experimental setup consisting of a Göttingen type wind tunnel with $u_{\infty}=10-20 \frac{\mathrm{m}}{\mathrm{s}}$, a thermography camera shown in red, and a DU95-W-180 airfoil mounted on a rotary table. Above the test section, the airfoil with the FOV of the thermographic camera is represented by an exemplary differential image thermogram.

determination of the temperature loss induced by the developing boundary layer flow.

To capture a broad range of boundary layer states different velocities from $u_{\infty}=10 \mathrm{~m} / \mathrm{s}$ to $u_{\infty}=20 \mathrm{~m} / \mathrm{s}$ are investigated. Further the AoA of the airfoil is set to $[0,5,10]^{\circ}$ successively. By this not only transition, but also the onset of a static stall can be observed during investigations.

\section{Thermography measurements}

To analyze a boundary layer flow, the different heat exchange properties between object surface and flow are used in DIT. These are characterized by the Stanton number defined as

$$
S t=\frac{h}{u \rho_{\text {Fluid }} c_{P}},
$$

where $h$ denotes the convection heat transfer coefficient, $u$ the flow velocity, $\rho_{\text {Fluid }}$ the density of the fluid and $c_{P}$ the specific heat of the fluid. In a laminar flow, $h$ and therefore $S t$ is significantly smaller compared to a turbulent flow as heat is dissipated slower ${ }^{28}$. Areas where the boundary layer is turbulent show increased cooling. Thus, the evolving temperature difference can be used to draw a direct conclusion on the flow state of the boundary layer.

To determine the transition point $\widetilde{R e_{c}}$ from the DIT measurements, the temperature gradient developing on the suction side of the airfoil is examined. Fig. 2 shows a color-coded representation of the measured time averaged differential temperatures. Each column represents an AoA. The rows represent different wind speeds. In addition, the temperature gradient averaged along the $\mathrm{y}$-direction is drawn as a red line for illustration purposes. The right ordinate of the pictures represent the temperature scale corresponding to the shown colors. At this point it should be noted that the temperature scale in Fig. 2(a) differs from the others, but covers the same temperature range.

The measured DITs can be divided into two groups. The first group shows a temperature drop and a minimum along the chord (Fig. 2 (a)-(c), (e), (f)), while the second group shows either a slowly decreasing temperature (Fig. $2(\mathrm{~d})$ ) or a monotonous increase (Fig. 2 (g)-(i)). Temperature gradients observed in the first group are typical for laminar-turbulent boundary layer transitions. The initial laminar flow and the related low $S t$ number result in a higher surface temperature. As the flow transitions to turbulence, $h$ and thus $S t$ increases, leading to a decrease in surface temperature. Using this information, the gradients can be utilized to determine the location of the transition point based on shown results.

First, the inflection point of each curve is calculated $\left(\min \left(\frac{d \Delta T}{d x}\right)\right)$. These points are shown in Fig. 2 as diamond symbols. As the transition between the two boundary layer states is not a step function, the points of maximum curvature are also determined $\left(\min \left(\frac{d^{2} \Delta T}{d x^{2}}\right)\right.$, $\left.\max \left(\frac{d^{2} \Delta T}{d x^{2}}\right)\right)$. These points are used to estimate the range in which the transition can take place. They are shown as crosses in Fig. 2. The transition points $\left(\widetilde{R e_{c}}\right)$ extracted from DIT images are summarized in Tab. 1, where $\Delta \widetilde{R e_{c}}$ represents the smallest difference to one of the upper bounds. The transition points are given in units of the chord length based Reynolds number defined as

$$
R e_{x}=\frac{u_{\infty} x}{\nu},
$$

where $x$ defines the position along the chord, $u_{\infty}$ the free stream velocity and $\nu$ the kinematic viscosity.

In contrast to $\alpha=0^{\circ}$, measurements at $\alpha=5^{\circ}$ show a 

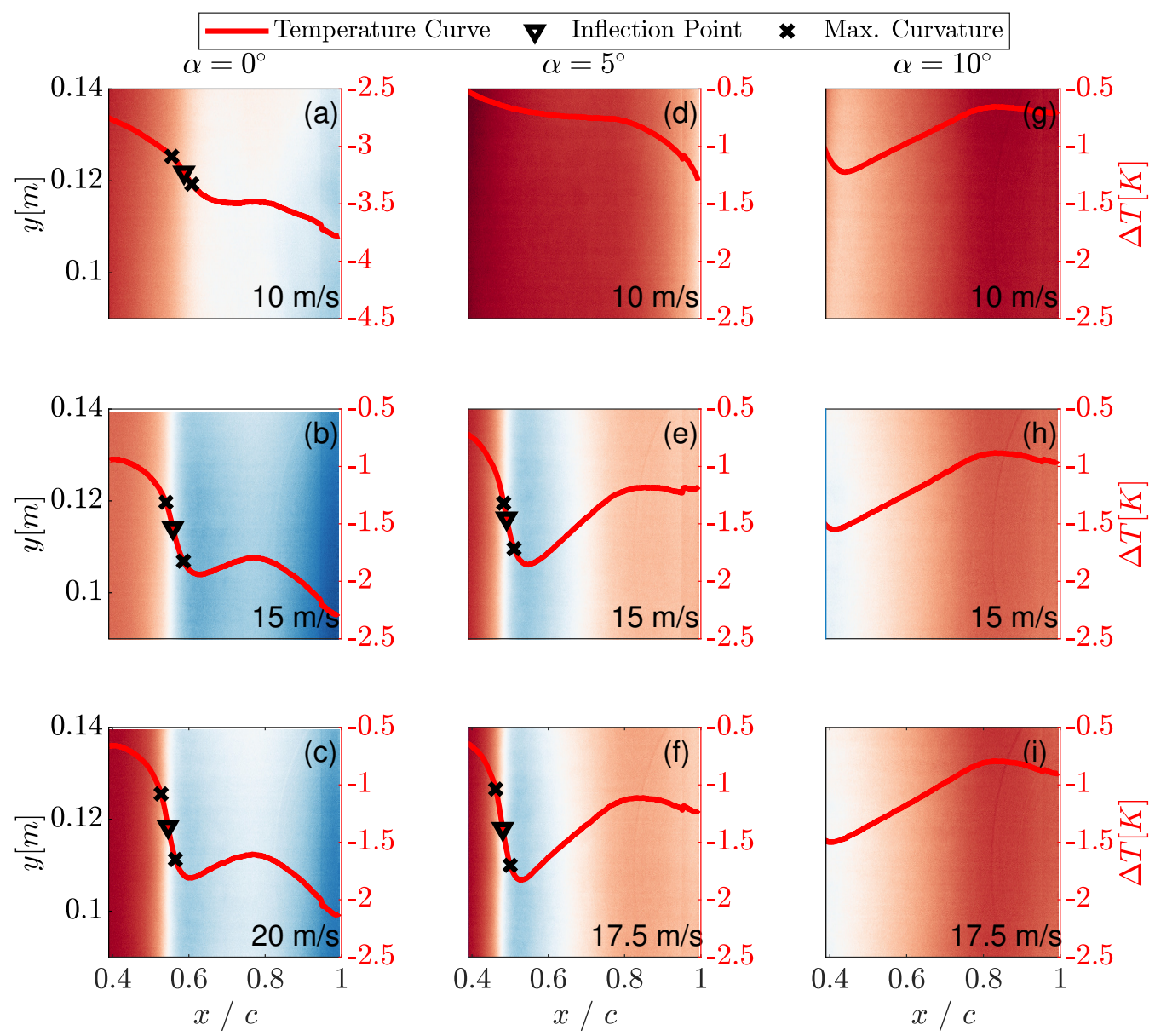

Figure 2. Time averaged differential image thermograms for different AoA $[0,5,10]^{\circ}$ and velocities from $10 \mathrm{~m} / \mathrm{s}$ $20 \mathrm{~m} / \mathrm{s}$. The colors represent the temperature from warm (red) to cold (blue). Additionally, the spatial averaged temperature curves are plotted in red. The black symbols represent the inflection point and the point of maximum curvature. The right-hand side ordinate corresponds to shown color code.

temperature rise at $x / c \approx 0.7$ (Fig. $2(\mathrm{e}),(\mathrm{f}))$. This is indicative of another flow phenomenon besides the transition from laminar to turbulent flow. The increasing temperature must be related to a decrease in $S t$ and thus decreased heat transfer. An obvious explanation is a stall cell evolving from the trailing edge leading to a decreased velocity at the airfoils surface, which agrees with the characterization of this type of airfoil in the literature ${ }^{33}$.

For the second group of measurements no temperature decrease can be observed. The slopes of the gradients indicate the transitions from laminar to turbulent flows occur upstream of the observed FOVs. Therefore, the transition point cannot be quantified in the just discussed way from the available DIT data. The transition point can only be specified as being in front of the FOV under consideration in Tab. 1. Besides the absence of the transition, however, the measurements at an AoA of $\alpha=$ $10^{\circ}$ also show the monotonic increase in the temperature gradient. This indicates that a stall also occurs here, but due to the larger AoA further upstream $(x / c \approx 0.5)$.

\section{Percolation theory}

Next, it is investigated from time-resolved DITs whether the laminar-turbulent transition fits to a universality class of directed percolation. Based on the results presented $\mathrm{in}^{20}$, the $(1+1) \mathrm{D}$ percolation is chosen for this investigation. Therefore, the measured DITs are first binarized and the turbulent fraction $\rho$ is determined. These data are used to check the agreement between universal exponents and experimental data.

In percolation theory, the cells within the system can only take two states, on or off. For a transient boundary layer this corresponds to turbulent (on) or laminar (off). The distribution of turbulent cells or the so-called turbulent fraction $\rho$ is described in percolation theory by an order parameter $p$. In the case of performed experiment, the 
Table 1. Transition point $\widetilde{R e_{c}}$ extracted from DIT measurements referring to Fig. 2.

\begin{tabular}{|c|c|c|c|}
\hline Measurement & $\widetilde{R e_{c}}$ & $\Delta \widetilde{R e_{c}}$ & $\Delta \widetilde{R e_{c}} / \widetilde{R e_{c}}$ \\
\hline (a) & 66909 & 2329 & $3.4 \%$ \\
\hline (b) & 95380 & 3105 & $3.3 \%$ \\
\hline (c) & 124283 & 4160 & $3.4 \%$ \\
\hline (d) & $<44049$ & - & - \\
\hline (e) & 83875 & 1331 & $1.6 \%$ \\
\hline (f) & 95836 & 3698 & $3.9 \%$ \\
\hline (g) & $<42151$ & - & - \\
\hline (h) & $<63465$ & - & - \\
\hline (i) & $<72551$ & - & - \\
\hline
\end{tabular}

chord length based Reynolds number $R e_{x}$ is appropriate (see Eqn. (2)). The following relation between turbulent fraction $\rho$ and order parameter $R e_{x}$ holds true:

$$
\rho\left(R e_{x}\right)=\rho_{0}\left(\frac{R e_{x}-R e_{c}}{R e_{c}}\right)^{\beta},
$$

which was already used in ${ }^{18,20} . R e_{c}$ is the critical Reynolds number defining where the transition between a non-percolating and a percolating system takes place. If the percolation can describe the transition location as well, this $R e_{c}$ should be like the $\widetilde{R e_{c}}$ from DIT. $\rho_{0}$ is a normalization factor. The exponent $\beta$ is the first of three universal exponents, which depends on the considered systems dimensions. For the $(1+1) \mathrm{D}$ directed percolation, the exponent is given by $\beta=0.276^{7}$. The directed percolation theory has, besides $\beta$, two additional universal exponents $\nu_{\perp}$ and $\nu_{\|}$. These exponents characterize the divergent behavior of the correlation length $\xi_{\perp, \|}$ in the different directions of the system when the critical point $R e_{c}$ is approached. For an airfoil where the order parameter changes along the chord, the correlation lengths refer to the y-direction and the time evolution and are described by the following relation:

$$
\xi_{\perp} \propto\left|R e-R e_{c}\right|^{\nu \perp}, \xi_{\|} \propto\left|R e-R e_{c}\right|^{\nu_{\|}} .
$$

From theory the exponents $\nu$ are given for the $(1+1) \mathrm{D}$ percolation with $\nu_{\perp,(1+1) D}=1.097$ and $\nu_{\|,(1+1) D}=$ $1.733^{7}$. With the help of the hyper scaling relation ${ }^{20}$, instead of the correlation length scales, also the cluster sizes of the on or off states can be considered, which scale with the exponent

$$
\mu_{\perp, \|}=2-\frac{\beta}{\nu_{\perp, \|}} .
$$

Based on available experimental data, it is more straightforward to determine the cluster sizes instead of using the correlation lengths, which is why this relationship is used in the following.
To determine the state of the boundary layer (laminar/turbulent) from the differential temperature measurements, each image needs to be binarized by a threshold. Temperatures above the threshold correspond to a laminar and below to a turbulent state. This threshold value equals the previously determined inflection point of the respective measurement (see Fig. 2 ). If no inflection point can be determined, the value at the left edge of the FOV is chosen, since the transition probably occurred upstream of the FOV. An example of a binarized thermogram is shown in Fig. 3.

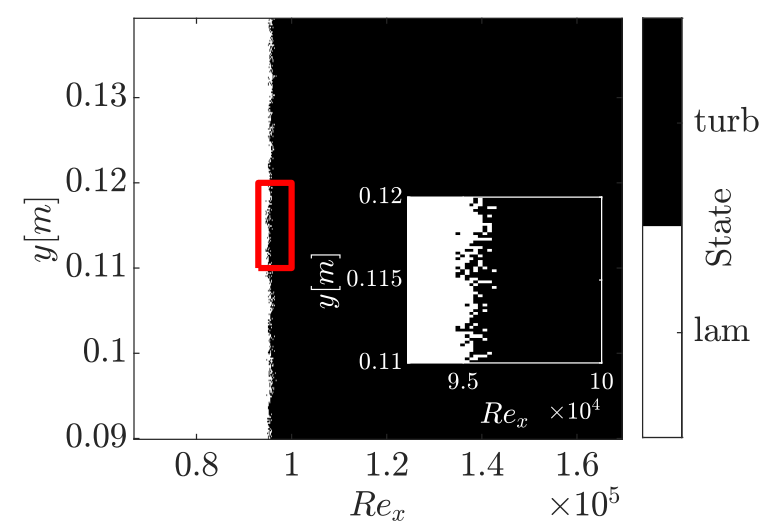

Figure 3. Binarized differential image thermogram of measurement Fig. 2 (b) using the defined threshold. Inlay shows a zoom into the transition area marked by the red box.

By averaging all binarized images of each measurement the turbulent fraction $\rho=\rho\left(R e_{x}, y\right)$ can be calculated. Fig. 4 shows $\rho$ for measurement Fig. 2 (b). The increase of the turbulent fraction from 0 to 1 is close to a step function and changes hardly in y-direction.

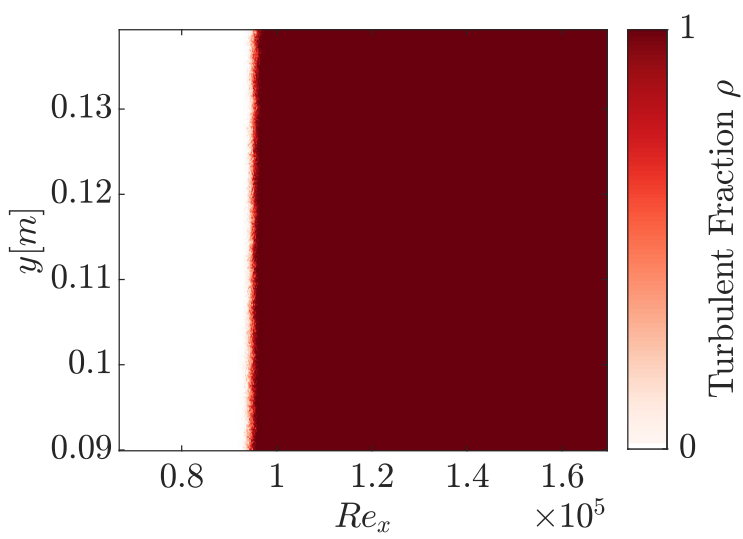

Figure 4. Turbulent fraction $\rho$ calculated from the binarized differential image thermograms of measurement Fig. 2 (b).

For further analysis, $\rho\left(R e_{x}, y\right)$ is averaged along the 
span wise direction (y-direction). The result is shown in Fig. 5 for the corresponding measurements of Fig. 2 (b),(e),(h) and (d). The figure shows $\rho$ over $R e_{x}$. The turbulent fraction indicates a very defined transition from a laminar, non-percolating system $(\rho=0)$ to a fully turbulent, percolating system $(\rho=1)$. For the cases (d) and (h) $\rho$ does not start at zero since the transition takes place upstream of the FOV.

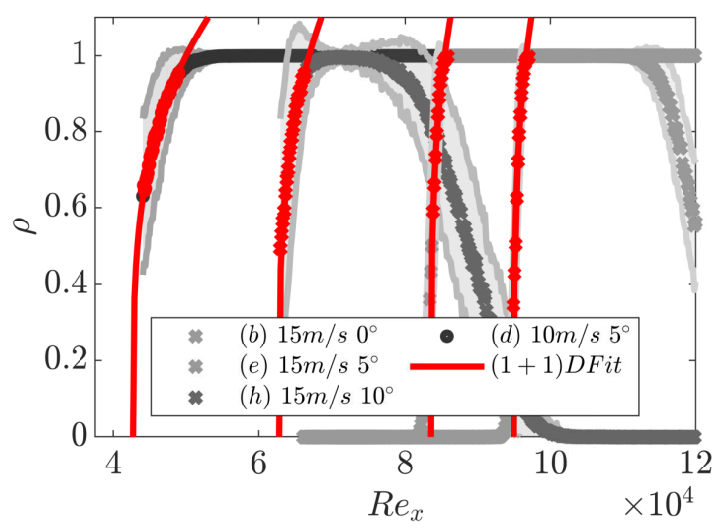

Figure 5. Turbulent fraction $\rho$ as result of taking the average along the y-direction from Fig. 4. Shown error bars represent the standard deviation of the averaging. Red curves show a fit using Eqn. (3). The red symbols on each curve represent the points used for fitting.

Besides the turbulent fraction Fig. 5 also contains the fits according to Eqn. (3) shown as red solid line. The red symbols of each curve indicate the points considered during the fitting routine, where $\beta$ is fixed at its theoretical value. To demonstrate alternatively the agreement of Eqn. (3), Fig. 6 shows the normalized turbulent fraction over $\epsilon=\frac{R e_{x}-R e_{c}}{R e_{c}}$ for all nine measurements. A clustering around the theoretical slope of $\beta=0.276$ is clearly seen.

The transition point $R e_{c}$ can be obtained from the fits as a fitting parameter. For all measurements the transition points are summarized in Tab. 2. The given error $\Delta R e_{c}$ is extracted as confident interval of $95 \%$ from the fit.

As another quantity the distribution of cluster sizes is investigated with respect to the universal exponents. Since the relations from Eqn. (4) are only valid at the critical point, the cluster sizes need to be extracted at determined $R e_{c}$ values. If $R e_{c}$ is outside the investigated FOV, as is the case for the measurements of group two (Fig. 2 (d),(g)-(i)), the measurements are excluded from the following analysis.

To determine the cluster sizes from the data, the binarized DITs are combined into a 3D matrix using the time as a third dimension resulting in a matrix with the dimensions $\left[R e_{x}, \mathrm{y}, \mathrm{t}\right]$. A cut is made through this matrix at $R e_{x}=R e_{c}$. This results in Fig. 7 containing

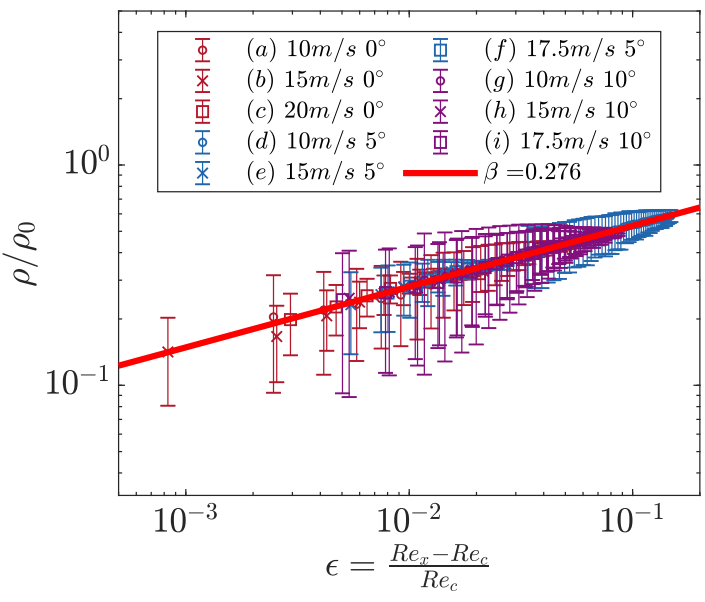

Figure 6. Logarithmic representation of the normalized turbulent fraction $\rho$ over $\epsilon=\frac{R e_{x}-R e_{c}}{R e_{c}}$ for all performed measurements. The red solid line represents the theoretical slope of $\beta$.

Table 2. Transition point extracted from $(1+1) \mathrm{D}$ directed percolation model fit like shown in Fig. 5.

\begin{tabular}{|c|c|c|c|}
\hline Measurement & $R e_{c}$ & $\Delta R e_{c}$ & $\Delta R e_{c} / R e_{c}$ \\
\hline (a) & 66192 & 47 & $0.07 \%$ \\
\hline (b) & 94794 & 53 & $0.06 \%$ \\
\hline (c) & 123918 & 169 & $0.13 \%$ \\
\hline (d) & 42706 & 77 & $0.18 \%$ \\
\hline (e) & 83583 & 164 & $0.19 \%$ \\
\hline (f) & 95458 & 200 & $0.21 \%$ \\
\hline (g) & 41778 & 87 & $0.21 \%$ \\
\hline (h) & 62781 & 50 & $0.08 \%$ \\
\hline (i) & 71589 & 60 & $0.08 \%$ \\
\hline
\end{tabular}

the laminar and turbulent spots in a $(\mathrm{y}, \mathrm{t})$ domain from which the cluster sizes can be determined in y-direction (spatial, $L_{s}$ ) and t-direction (temporal, $L_{t}$ ).

Fig. 8 shows the resulting cluster size distributions in spatial direction with the theoretically expected slope of $\mu_{\perp}=1.748$ for conducted measurements. $N_{s}$ is the number of clusters of a given size $L_{s}$. The cluster size distributions clearly follow the theoretical value. This can be emphasized especially by the mean value of all cluster distributions shown as black line. The reason for the noise occurring with large clusters is the rare appearance of such large structures in the system and the resulting higher statistical uncertainty.

The temporal cluster size distributions of the parallel direction are shown in Fig. 9. Again, the distributions are shown together with the theoretical value of $\mu_{\|}=1.84$. Like the spatial cluster size distributions, the temporal cluster size distributions fit very well to the 


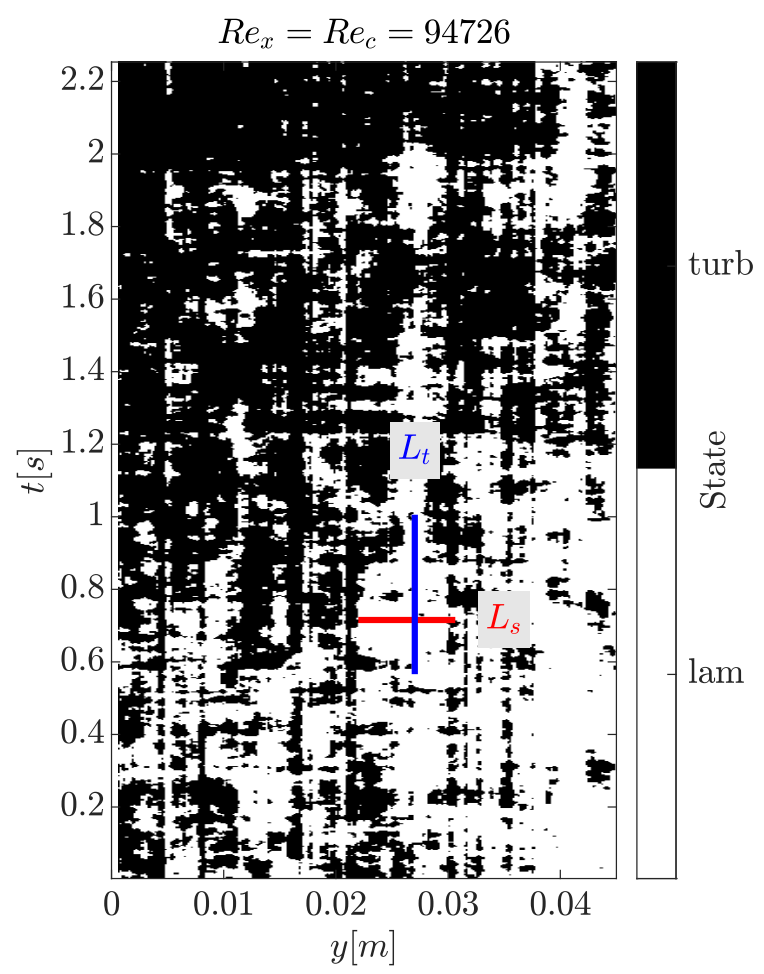

Figure 7. Exemplary representation of the definition of cluster sizes in spatial $\left(L_{s}\right)$ and temporal $\left(L_{t}\right)$ direction. This figure is based on measurement (b).

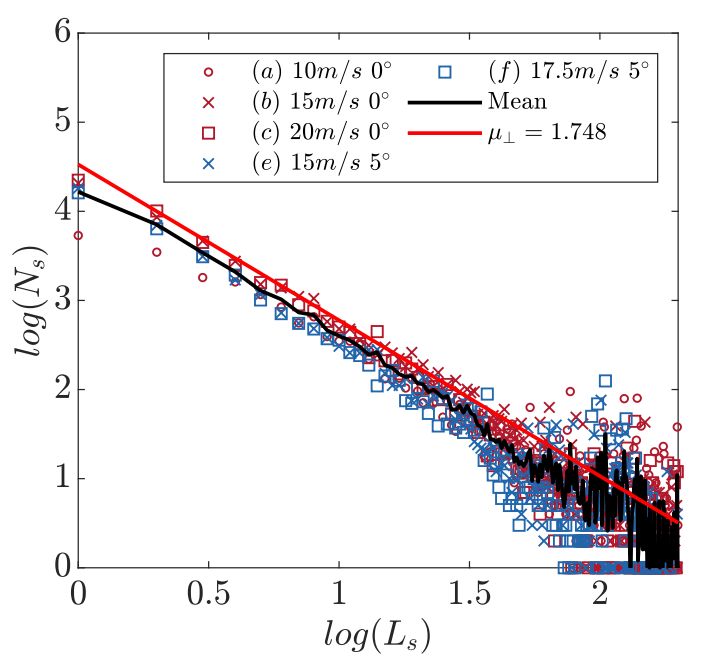

Figure 8. Cluster size distribution for spatial cluster sizes $(\perp)$ for all measurements. The black line represents the mean of all measurements. The red line indicates the theoretical slope for $(1+1) \mathrm{D}$ directed percolation.

theoretically expected value. Even for the large cluster sizes the measurements are in good agreement with theory.

We remark that this power law behavior of the cluster sizes will change sensitively as chosen $R e_{x}$ differs from $R e_{c}$

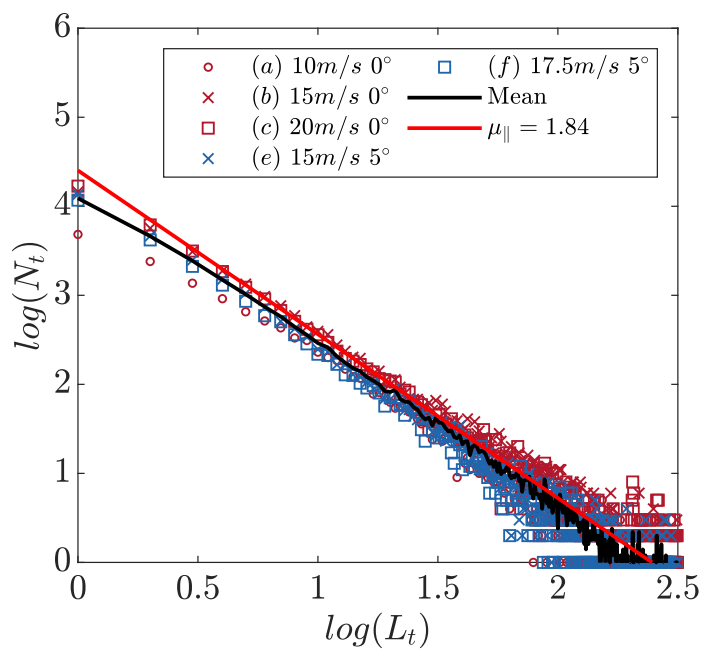

Figure 9. Cluster size distribution for temporal cluster sizes $(\|)$ for all measurements. The black line represents the mean of all measurements. The red line indicates the theoretical slope for $(1+1) \mathrm{D}$ directed percolation.

\section{Summary and Conclusion}

In this study the near surface flow of an airfoil is investigated for a large range of velocities and angle of attacks. Differential image thermography (DIT) is used to access the boundary layer state indirectly by using the heat transfer properties of different flow situations. DIT offers the advantage of being able to measure along curved surfaces, as their temperature is recorded directly. Thus, both the laminar-turbulent transition of the developing boundary layer and the onset of flow separation from the trailing edge can be captured by the DIT from presented measurements.

This first part of the study shows the potential of the DIT in the field of aerodynamic investigations. Despite the very simple setup, profound information about the state of the boundary layer flow can be extracted and different states can be distinguished. The location of the transition was determined, but only with a limited accuracy due to the shape of the temperature gradient of the raw data (see Tab. 1). Furthermore, the determination is only possible if the entire transition is taking place in the FOV of the measurement.

Based on preliminary evidence that the transition from laminar to turbulent flow can be described by $(1+1) \mathrm{D}$ directed percolation, the DIT data are further evaluated using this theory. Here, a good agreement of the measured data with the universal exponents is found for a large range of velocities and angle of attacks. The 
relationship between the turbulent fraction and order parameter described by the universal exponent $\beta$ is further used to calculate the critical Reynolds number $R e_{c} . R e_{c}$ corresponds to the transition point and can be determined much more precisely by this evaluation compared to the DIT. The relative accuracy is improved by more than one order of magnitude (see Tab. 2). Furthermore, this theoretical relationship can be used to determine transition points outside the FOV.

In addition to the first universal exponent $\beta$, the remaining two exponents $\mu_{\perp, \|}$ are also examined. Their theoretical slopes fit very well to the measured data, which further supports the assumption that the $(1+1) \mathrm{D}$ percolation can generally describe the transition process. It should be noted that there is no experimental study so far that has been able to show the agreement of measured data with the exponent $\mu_{\|}$over such a large range. Moreover, the agreement could mostly be demonstrated only for generic flows and simulations.

Overall, this study shows how directed percolation theory can be used as a high precision tool to capture and describe the transition of a boundary layer. The agreements found for all three exponents clearly show that the transition of an airfoil boundary layer can be assigned to the $(1+1) \mathrm{D}$ universal class with very high probability. This is emphasized by the fact that the exponents agree for a wide range of experimental conditions $\left(u_{\infty}, \alpha\right)$.

Based on these findings, percolation is suitable as a simple model for the highly dynamic and nonlinear processes in the boundary layer and integrated into CFD models to simulate them. By reducing the process to only three exponents and making it applicable to real-world problems, such as airfoils, this would also have far-reaching benefits for industrial applications (e.g., wind turbine modeling).

\section{Acknowledgements}

The authors would like to expressly thank Enno Bösenberg, who was substantially helping during in the measurements.

We would also like to thank Pedro Lind for fruitful and helpful discussions during data analysis and interpretation.

In addition we acknowledge the Lower Saxony Ministry of Science and Culture (MWK) for financial support of this study.

\section{References}

1. Reynolds, O. Xxix. an experimental investigation of the circumstances which determine whether the motion of water shall be direct or sinuous, and of the law of resistance in parallel channels. Philos. Transactions Royal society Lond. 935-982 (1883).
2. Manneville, P. Transition to turbulence in wallbounded flows: Where do we stand? Mech. Eng. Rev. 15-00684 (2016).

3. Barkley, D. Theoretical perspective on the route to turbulence in a pipe. J. Fluid Mech. 803 (2016).

4. Pomeau, Y. Front motion, metastability and subcritical bifurcations in hydrodynamics. Phys. D: Nonlinear Phenom. 23, 3-11 (1986).

5. Chaté, H. \& Manneville, P. Transition to turbulence via spatio-temporal intermittency. Phys. review letters 58, 112 (1987).

6. Rupp, P., Richter, R. \& Rehberg, I. Critical exponents of directed percolation measured in spatiotemporal intermittency. Phys. Rev. E 67, 036209 (2003).

7. Hinrichsen, H. Non-equilibrium critical phenomena and phase transitions into absorbing states. Adv. physics 49, 815-958 (2000).

8. Pomeau, Y. The long and winding road. Nat. Phys. 12, 198-199 (2016).

9. Allhoff, K. T. \& Eckhardt, B. Directed percolation model for turbulence transition in shear flows. Fluid Dyn. Res. 44, 031201 (2012).

10. Sipos, M. \& Goldenfeld, N. Directed percolation describes lifetime and growth of turbulent puffs and slugs. Phys. Rev. E 84, 035304 (2011).

11. Kreilos, T. et al. Bypass transition and spot nucleation in boundary layers. Phys. Rev. Fluids 1, 043602 (2016).

12. Rehill, B. et al. Identifying turbulent spots in transitional boundary layers. J. turbomachinery $\mathbf{1 3 5}$ (2013).

13. Barkley, D. Simplifying the complexity of pipe flow. Phys. Rev. E 84, 016309 (2011).

14. Shih, H.-Y., Hsieh, T.-L. \& Goldenfeld, N. Ecological collapse and the emergence of travelling waves at the onset of shear turbulence. Nat. Phys. 12, 245-248 (2016).

15. Avila, M. \& Hof, B. Nature of laminar-turbulence intermittency in shear flows. Phys. Rev. E 87, 063012 (2013).

16. Barkley, D. et al. The rise of fully turbulent flow. Nature 526, 550-553 (2015).

17. Lemoult, G. et al. Directed percolation phase transition to sustained turbulence in couette flow. Nat. Phys. 12, 254-258 (2016).

18. Sano, M. \& Tamai, K. A universal transition to turbulence in channel flow. Nat. Phys. 12, 249-253 (2016). 
19. Wester, T. T., Traphan, D., Gülker, G. \& Peinke, J. Percolation: statistical description of a spatial and temporal highly resolved boundary layer transition. In Progress in Turbulence VII, 11-16 (Springer, 2017).

20. Traphan, D., Wester, T. T., Gülker, G., Peinke, J. \& Lind, P. G. Aerodynamics and percolation: Unfolding laminar separation bubble on airfoils. Phys. Rev. X 8, 021015 (2018).

21. Manneville, P. \& Shimizu, M. Subcritical transition to turbulence in wall-bounded flows: the case of plane poiseuille flow. arXiv preprint arXiv:1904.03739 (2019).

22. Lemoult, G. et al. An experimental approach to directed percolation in pipe ow. Bull. Am. Phys. Soc. (2020).

23. Klotz, L., Lemoult, G. \& Hof, B. Couette flow transition in $2 \mathrm{~d}$ directed percolation universality class. Bull. Am. Phys. Soc. (2020).

24. Shih, H.-Y. et al. Directed percolation and puff crystallization near the transition to pipe turbulence. Bull. Am. Phys. Soc. (2020).

25. Raffel, M. \& Merz, C. B. Differential infrared thermography for unsteady boundary-layer transition measurements. AIAA journal 52, 2090-2093 (2014).

26. Gardner, A., Eder, C., Wolf, C. \& Raffel, M. Analysis of differential infrared thermography for boundary layer transition detection. Exp. Fluids 58, 122 (2017).

27. Wolf, C. C., Mertens, C., Gardner, A. D., Dollinger, C. \& Fischer, A. Optimization of differential infrared thermography for unsteady boundary layer transition measurement. Exp. Fluids 60, 19 (2019).

28. Raffel, M., Merz, C. B., Schwermer, T. \& Richter, K. Differential infrared thermography for boundary layer transition detection on pitching rotor blade models. Exp. Fluids 56, 30 (2015).

29. Gardner, A. D., Wolf, C. C. \& Raffel, M. A new method of dynamic and static stall detection using infrared thermography. Exp. Fluids 57, 149 (2016).

30. Raffel, M. et al. Rotating blade stall maps measured by differential infrared thermography. AIAA J. 55, 1753-1756 (2017).

31. Reichstein, T. et al. Investigation of laminarturbulent transition on a rotating wind-turbine blade of multimegawatt class with thermography and microphone array. Energies 12, 2102 (2019).

32. Dollinger, C., Sorg, M., Balaresque, N. \& Fischer, A. Measurement uncertainty of ir thermographic flow visualization measurements for transition detection on wind turbines in operation. Exp. Therm. Fluid Sci. 97, 279-289 (2018).
33. Timmer, W. \& Van Rooij, R. Summary of the delft university wind turbine dedicated airfoils. J. Sol. Energy Eng. 125, 488-496 (2003).

\section{Author contributions}

All authors contributed to the study conception and design. Material preparation, data collection and analysis were performed by Tom T. B. Wester. Joachim Peinke and Gerd Gülker have contributed significantly to the development of the results through discussions. The first draft of the manuscript was written by Tom T. B. Wester and all authors commented on previous versions of the manuscript. All authors read and approved the final manuscript.

\section{Competing interests}

The authors declare no competing interests. 